\title{
STRA6 Gene
}

National Cancer Institute

\section{Source}

National Cancer Institute. STRA6 Gene. NCI Thesaurus. Code C104693.

This gene is involved in organogenesis. 